\title{
Effects of the environment on the properties of Seyfert galaxies
}

\section{Marcio A. G. Maia ${ }^{1,2}$, Christopher N. A. Willmer ${ }^{2,3}$, Bruno M. Rossetto ${ }^{1}$ and Rodolfo S. Machado ${ }^{1}$}

1 Observatório do Valongo, Universidade Federal do Rio de Janeiro, Ladeira do Pedro Antônio 43, Rio de Janeiro - 20080-090, RJ, Brazil

${ }^{2}$ Observatório Nacional, Rua Gal. José Cristino 77, Rio de Janeiro - 20921-900, RJ, Brazil

${ }^{3}$ Lick Observatory, University of California, Santa Cruz, 1156 High Street, Santa Cruz, CA 95064, USA

\begin{abstract}
We identify 175 Seyfert galaxies from the Southern Sky Redshift Survey - SSRS2. We use the entire SSRS2 catalog to investigate the correlation between the presence of AGN with host environment. The AGN phenomenon is more strongly correlated with the internal host properties than with the external environment. In particular, we find that Seyferts reside in more luminous galaxies, and are twice as frequent in barred galaxies and systems showing signs of advanced merger condition, when compared to a control sample.
\end{abstract}

\section{Introduction and Sample Definition}

In order to study the dependence of AGN properties with local host environment and to derive reliable statistical results (see contribution of $\mathrm{H}$. Schmitt in these proceedings), it is extremely important to select a suitable control sample. In this work we use the SSRS2 catalog of da Costa et al. (1998), which contains 5399 galaxies with $m_{B} \leqslant 15.5$ covering 1.69 sr of the southern sky. This catalog is currently $99.99 \%$ complete in redshift, and in our database we have optical spectra available for $\sim 78 \%$ of the SSRS2 galaxies, while the remaining radial velocities were obtained from the literature. The spectra were inspected and AGNs identified using the diagnostic diagrams of line intensity ratios proposed by Baldwin, Phillips \& Terlevich (1981). New Seyfert galaxies were detected during the survey and were reported in papers by Maia et al. (1987 and 1996). Additional AGN hosts in the SSRS2 were obtained consulting the NASA Extragalactic Database, and the literature. For AGN host galaxies without spectra in the SSRS2 database, new observations were taken, therefore keeping the identification process homogeneous. Our AGN sample is made up of 175 Seyferts, which comprise $3-4 \%$ of the parent sample. The ratio Sey-2/Sey-1 is 3:1. This fraction may contain an excess of Sey-2 which are in reality Sey-1 with broad lines that are partially obscured by the material in the disk of host galaxies seen almost edge-on.

\section{Internal Properties of Seyfert Hosts}

We first examine the distribution of morphologies of the Seyfert galaxy population, which is displayed in figure 1. Also in this figure is shown the morphological distribution of the SSRS2 parent catalog. Seyfert galaxies are distributed preferentially among the S0a$\mathrm{Sb}$ interval and in those hosts showing strong evidence of mergers (type 15). In particular, the excess of Seyferts in type 15, could be an indication that strong interactions may 


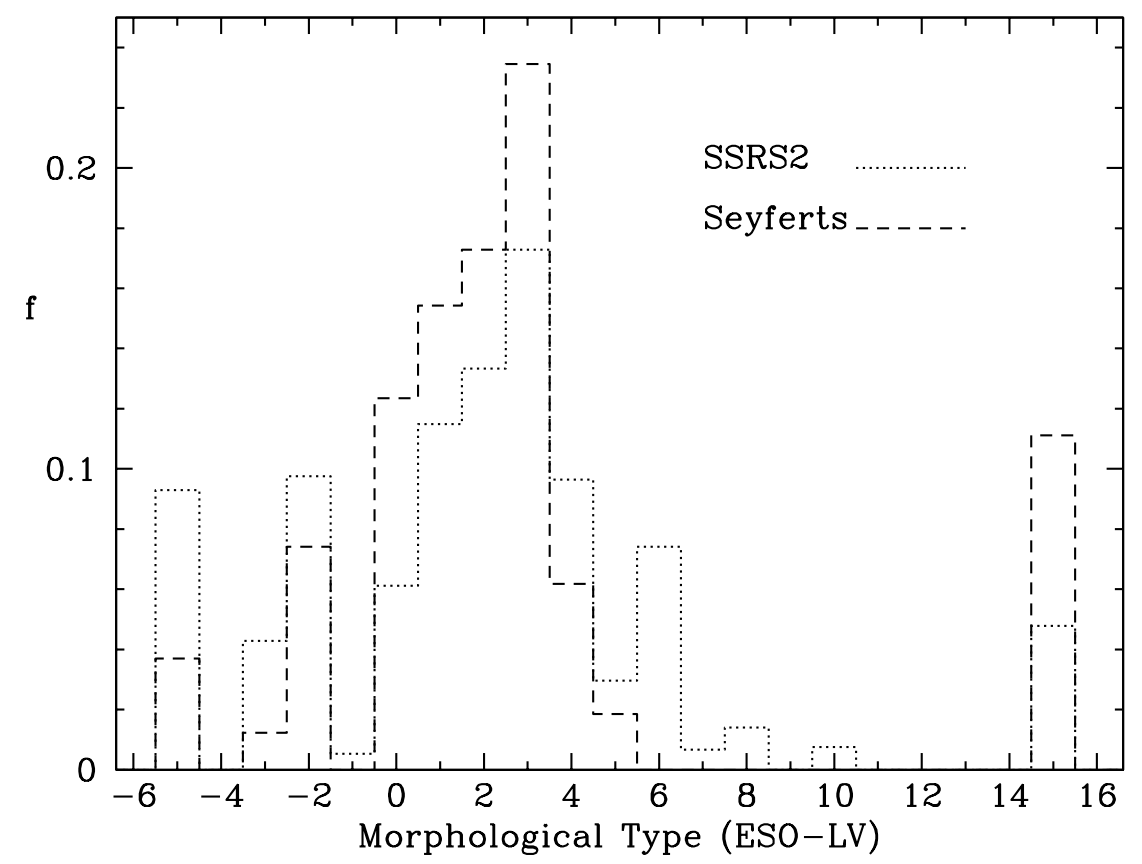

Figure 1. Morphological distribution of Seyferts and SSRS2 galaxies. The numbers correspond to the following classification: $\mathrm{E}=-5, \mathrm{E} / \mathrm{S} 0=-3, \mathrm{~S} 0=-2, \mathrm{~S} 0 \mathrm{a}=0, \mathrm{Sa}=1, \mathrm{Sab}=2, \mathrm{Sb}=3, \mathrm{Sbc}=4$, $\mathrm{S} \ldots=5, \mathrm{Sc}=6, \mathrm{Sc} / \mathrm{Irr}=7, \mathrm{Sd}=8, \mathrm{Irr}=10$, merger $=15$.

trigger or enhance the nuclear activity. No significant difference was seen between the host morphologies of Seyfert types 1 and 2.

We also investigated the presence of bars, which have been claimed as a possible mechanism to induce gas and dust radial inflows, fueling the AGN activity. We inspected visually DSS images of the entire SSRS2 catalog members. Because of saturation in the central parts of galaxy images in the DSS, the presence of bars cannot be identified for many galaxies, while for galaxies that are edge-on or which have small apparent sizes bars are also hard to recognize. Nevertheless, we are able to detect, at least, the relative differences between the Seyferts and control samples. Globally, we find that the SSRS2 contains $14 \%$ barred galaxies, while the Seyfert sample contains $28 \%$. The fraction of barred galaxies as a function of morphological type, for the SSRS2 and Seyfert samples, is displayed in figure 2. There are twice as many barred hosts in the Seyfert population than in the SSRS2 control sample. This result is maintained even if we consider only the morphological types where Seyferts are more frequent. This fraction is the same for subsamples limited at different distances.

We examined the total luminosity distributions of the AGNs and SSRS2 samples. We find that Seyferts are prone to reside in more luminous hosts, when the ratio between the number densities per magnitude bin of Seyfert hosts and SSRS2 objects are computed.

\section{The External Environment of Seyfert Galaxies}

We examine the possible correlation between the presence of AGN phenomenon with the local density of galaxies by identifying groups of galaxies in the SSRS2. These groups are generated using a percolation algorithm analogous to that described by Merchán, 


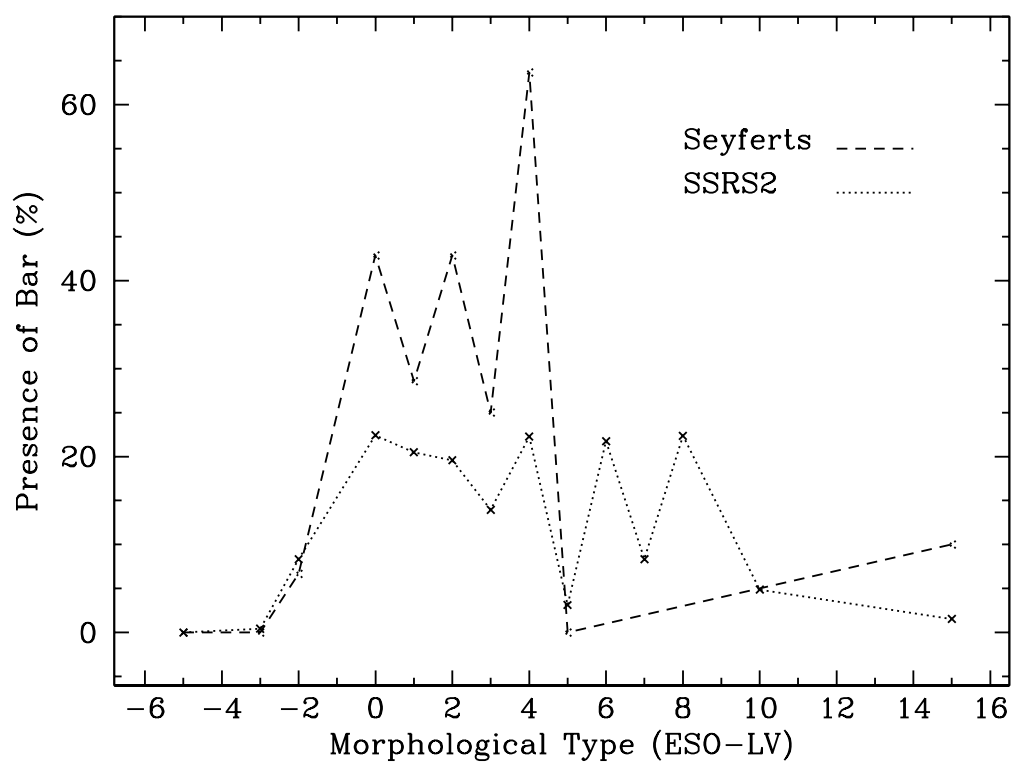

Figure 2. Fraction of galaxies with bars in the Seyfert and SSRS2 samples as a function of morphological type, using the same coding as in figure 1 .

Table 1. SSRS2 and Seyfert galaxies versus environment assignment

$\begin{array}{lcccc}\text { Galaxies } & \begin{array}{c}\text { Groups } \\ (\%)\end{array} & \begin{array}{c}\text { Triplets } \\ (\%)\end{array} & \begin{array}{c}\text { Binaries } \\ (\%)\end{array} & \begin{array}{c}\text { Isolated } \\ (\%)\end{array} \\ \text { Seyferts } & 21 & 7 & 28 & 44 \\ \text { SSRS2 } & 24 & 8 & 18 & 50\end{array}$

Maia, \& Lambas (2000). The algorithm also outputs a list of objects for which no companions are found ("isolated galaxies"). The assignments for Seyfert and SSRS2 samples according to different degrees of multiplicity are displayed in table 1. Both samples are distributed in a similar way, except for binary systems, in which Seyfert hosts are more commonly found when compared to other galaxies of the entire SSRS2. This suggests that galaxies undergoing close interactions could have the AGN phenomenon intensified.

We calculate the average group density, $\rho_{g}$, for both the AGN and SSRS2, by means of the expression $\rho_{g}=3 N_{g} / 4 \pi r^{3}$ where, $N_{g}$ is the number of galaxies and $r$ the mean pairwise separation of a given group. In figure 3 we display the median values for $\rho_{g}$ as a function of group multiplicity. There is no evidence of the AGN phenomenon to be correlated with group density.

Some results in the literature show evidence that the presence of a close companion may be correlated to the AGN activity. We tested this hypothesis by computing the nearest neighbor distance - $S$, and the maximum tidal influence - $Q$ that a companion may exert. The tidal influence is proportional to the companion's mass divided by the cube of the separation, $S$. Assuming that light traces mass, the tidal parameter is $Q \propto L / S^{3}$. The distribution of the nearest neighbor separation, $S$, for Seyferts and SSRS2 galaxies was examined using the KS test which shows that both samples are similar at the $98 \%$ confidence level. The distribution of $Q$ values for the AGNs and SSRS2 are identical at the 


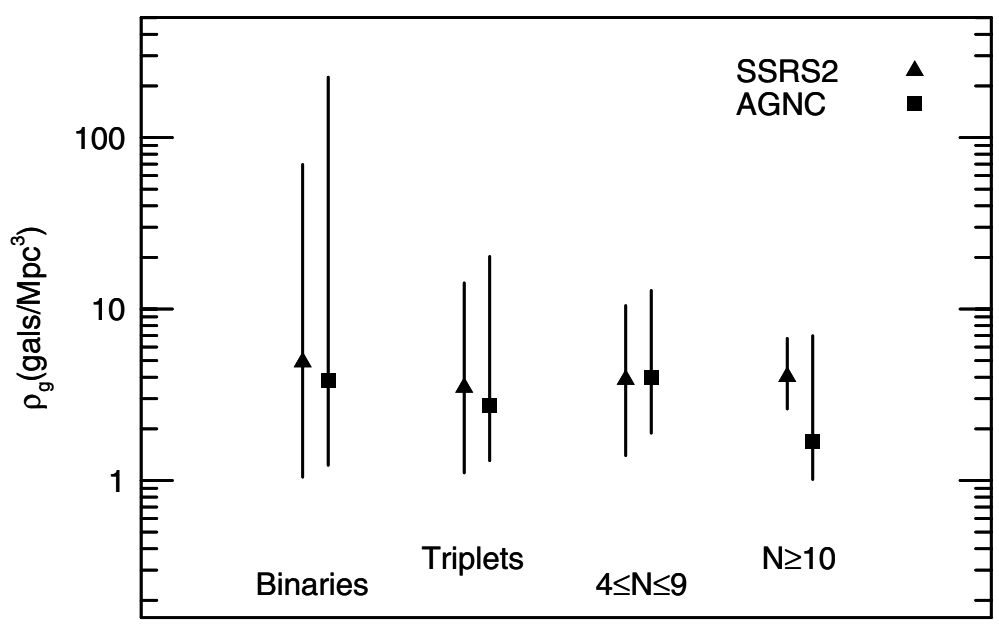

Multiplicity

Figure 3. Group density $\rho_{g}$, according to multiplicity intervals. The points represent median values, while the bars refer to upper and lower quartiles of the distributions.

$86 \%$ confidence level. Meanwhile, the Seyfert types 1 and 2 distributions are considered the same only at the $20 \%$ confidence level, with type 1 s presenting higher values of $Q$.

\section{Conclusions}

We used the magnitude-limited SSRS2 to identify Seyfert galaxies, and investigated how the properties of their hosts relate to general population of non-active galaxies. We find that $175 / 5339(3-4 \%)$ of the galaxies in the SSRS2 are Seyferts. The ratio of Seyfert $2 \mathrm{~s}$ to $1 \mathrm{~s}$ is $\sim 3: 1$. Most Seyferts are of morphological types betwen S0a and Sb, and $\sim 10 \%$ have hosts with indication of an ongoing merger. There is no difference between the morphology of Seyfert types 1 and 2. The AGNs are preferentially detected in high luminosity hosts, and are twice as frequent in barred hosts. No correlation with local density of galaxies. We find marginal evidence that Seyfert 1 galaxies have closer companions and/or are more susceptible to tidal effects than type 2. Additional details about the results presented here can be obtained in the paper by Maia, Machado, \& Willmer (2003).

\section{References}

Baldwin, J. A., Phillips, M. M., \& Terlevich, R. 1981, PASP, 93, 5

da Costa, L. N., et al. 1998, AJ, 116, 1

Maia, M. A. G., da Costa, L.N., Willmer, C. N. A., Pellegrini, P. S., \& Rite, C. 1987, AJ, 93, 546

Maia, M. A. G., Machado, R. S., \& Willmer, C. N. A. 2003, AJ, 126, 1750

Maia, M. A. G., Suzuki, J. A., da Costa, L. N., Willmer, C. N. A., \& Rite, C. 1996, A\&A, 117, 487

Merchán, M. E., Maia, M. A. G., \& Lambas, D. G. 2000, ApJ, 545, 26 